Antonio M. Esquinas

Rachael Parke

Alex H. Gifford

\section{Failure of high-flow nasal cannula and delayed intubation: a new harmful sequence?}

Accepted: 4 April 2015

Published online: 24 April 2015

(C) Springer-Verlag Berlin Heidelberg and ESICM 2015

Dear Editor,

Presently, there is general agreement regarding the benefits of high-flow nasal cannula (HFNC) in certain forms of acute hypoxemic respiratory failure (AHRF); however, which clinical circumstances constitute HFNC failure and whether HFNC use unnecessarily delays endotracheal intubation (ETI) are controversial [1]. Kang et al. [3] analysed the impact of the timing of ETI relative to the determination of HFNC failure. We feel that this estimation warrants consideration.

First, from a methodological standpoint, Kang et al. [3] enrolled patients with multiple reasons for AHRF. Defining two cohorts on the basis of early or late ETI but having those cohorts contain different numbers of patients with each etiologic category of AHRF makes it difficult to determine the independent contribution of timing of ETI to the outcomes of interest. Indeed, we might expect patients with specific conditions, like haematological malignancies, to fare worse in the setting of AHRF, regardless of the decision to treat with invasive or non-invasive ventilation, than those with another diagnosis. Moreover, it is difficult to reconcile the comparison of patients with chronic obstructive pulmonary disease (COPD) to those with other categories of AHRF because of fundamental differences in pulmonary mechanics and gas exchange abnormalities [2].

To adjust for confounding factors and heterogeneity in baseline attributes of patients in their study, Kang et al. [3] use propensity score analyses to compare early and late ETI groups. This strategy is essentially a form of multivariate adjustment for covariates and has the advantage of identifying two groups with similar baseline characteristics, which facilitates their comparison on the basis of an endpoint, like timing of ETI. However, the process of matching patients with similar propensity scores can significantly reduce sample size, statistical power to discern differences in risk for the endpoint of interest, and external validity [4]. Indeed, Kang et al. were left with 37 patients in each group after matching by propensity score. Propensity score analyses also do not account for some forms of bias, including confounding by indication.

The authors described their institutional practice of monitoring patients on the general wards by a dedicated medical emergency team (MET). We think that this approach to patients with non-cardiogenic causes of AHRF (i.e. acute respiratory distress syndrome, pneumonia) is not particularly generalizable for two reasons. First, many hospitals cannot dedicate an intensivist, a senior resident and two nurses to this undertaking. Second, the study could not control for unknown biases that MET groups could have had for or against ICU transfer and/or the timing of ETI. It would be interesting to know whether ICU admission criteria used by MET groups evolved during observation period [5].

We also have concerns that the authors incorporated a need for ETI into their definition of HFNC failure. It would be instructive to know in greater detail about clinical conditions associated with early and late HFNC failure.

Finally, regarding the cohort of late HFNC failures, there are no clear reasons given for delayed ETI (refractory hypoxaemia, neurological impairment and/or haemodynamic instability). Further prospective clinical trials are needed to evaluate especially those that inappropriately delay ETI outside the ICU.

\section{References}

1. Ricard JD (2012) High flow nasal oxygen in acute respiratory failure. Minerva Anestesiol 78:836-841

2. Depuydt PO, Benoit DD, Vandewoude $\mathrm{KH}$, Decruyenaere JM, Colardyn FA (2004) Outcome in noninvasively and invasively ventilated hematologic patients with acute respiratory failure. Chest 126:1299-1306

3. Kang BJ, Koh Y, Lim CM, Huh JW, Baek S, Han M, Seo HS, Suh HJ, Seo GJ, Kim EY, Hong SB (2015) Failure of high-flow nasal cannula therapy may delay intubation and increase mortality. Intensive Care Med 41:623-632. doi: 10.1007/s00134-015-3693-5

4. Freemantle N, Marston L, Walters K, Wood J, Reynolds MR, Petersen I (2013) Making inferences on treatment effects from real world data: propensity scores, confounding by indication, and other perils for the unwary in observational research. BMJ 11(347):f6409. doi: 10.1136/bmj.f6409

5. Lovett PB, Massone RJ, Holmes MN, Hall RV, Lopez BL (2014) Rapid response team activations within 24 hours of admission from the emergency department: an innovative approach for performance improvement. Acad Emerg Med 21:667-672

A. M. Esquinas (-)

Intensive Care Unit, Hospital Morales Meseguer, Avenida Marques de Los Velez s/n, 30008 Murcia, Spain e-mail: antmesquinas@gmail.com

Tel.: (34) 609321966

R. Parke

Cardiothoracic and Vascular ICU, Auckland City Hospital, Auckland, New Zealand

A. H. Gifford

Section of Pulmonary and Critical Care Medicine, Dartmouth-Hitchcock Medical Center, Lebanon, New Hampshire, USA 